\title{
Cetuximab plus FOLFOX-4 in Untreated Patients with Advanced Colorectal Cancer: A Gruppo Oncologico dell'Italia Meridionale Multicenter Phase II Study
}

\author{
Giuseppe Colucci $^{a} \quad$ Francesco Giuliani $^{\mathrm{a}}$ Carlo Garufic ${ }^{c}$ Rodolfo Mattiolie ${ }^{\text {Luigi Manzione }}{ }^{f}$ \\ Antonio Russo $^{9}$ Massimo Lopez ${ }^{\mathrm{d}}$ Paola Parrella ${ }^{\text {h }}$ Stefania Tommasi ${ }^{b}$ Massimiliano Copetti ${ }^{i}$ \\ Bruno Daniele $^{k}$ Salvatore Piscontil Guido Tuveri $^{m}$ Nicola Silvestris ${ }^{a}$ Evaristo Maiello ${ }^{j}$

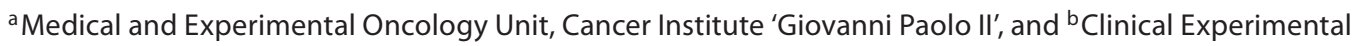 \\ Oncology Laboratory, Oncology Institute, Bari, Medical Oncology Units ${ }^{\mathrm{C}} \mathrm{C}$ and ${ }^{\mathrm{d}} \mathrm{B}$, Oncology Institute Regina Elena, \\ Rome, ${ }^{e}$ Medical Oncology Unit, General Hospital, Fano, ${ }^{f}$ Medical Oncology Unit, S. Carlo Hospital, Potenza, \\ 9 Interdepartmental Center of Research in Clinical Oncology, University of Palermo, Palermo, \\ ${ }^{\mathrm{h}}$ Oncology Laboratory and 'Biostatistics Unit, IRCCS Casa Sollievo della Sofferenza, and ${ }^{\mathrm{j}}$ Medical Oncology Unit, \\ Hospital Casa Sollievo della Sofferenza, San Giovanni Rotondo, ${ }^{k}$ Medical Oncology Unit, General Hospital, Benevento, \\ 'Oncology Unit, SS Annunziata Hospital, Taranto, and ${ }^{\mathrm{m}}$ Medical Oncology Unit, General Hospital, Trieste, Italy
}

\section{Key Words}

Colorectal carcinoma - Metastases - Cetuximab •

FOLFOX-4 $\cdot$ KRAS $\cdot$ BRAF

\begin{abstract}
Objectives: FOLFOX-4 and FOLFIRI are considered equivalent in terms of activity and efficacy as first-line chemotherapy in metastatic colorectal cancer ( $\mathrm{mCRC}$ ). The monoclonal antibody (mAb) cetuximab showed intrinsic activity as a single agent in $\mathrm{MCRC}$ and was approved in combination with CPT-11 for patients who failed previous CPT-11-based treatment. The purpose of this phase II study was to evaluate the activity and safety of FOLFOX-4 plus cetuximab in untreated mCRC patients. Methods: Untreated patients with measurable metastatic disease and expressing epidermal growth factor receptor (EGFR) received cetuximab at a loading dose of $400 \mathrm{mg} / \mathrm{m}^{2}$, followed by weekly doses of $250 \mathrm{mg} / \mathrm{m}^{2}$, in combination with the FOLFOX-4 regimen every 2 weeks for
\end{abstract}

a maximum of 12 cycles, after which a maintenance program using cetuximab alone was allowed for a maximum of 6 months. Results: Eighty-two unselected patients were screened; 70 were EGFR+ and entered the trial. Of the 67 assessable patients, the objective response rate was $64.2 \%$ (95\% Cl: 52.5-75.5\%) and the tumor growth control rate was 94\% (95\% Cl: 88-99\%). All the objective responses except 1 were confirmed. In the group of patients with initially unresectable liver disease alone, 7/33 (21\%) were resected. The median time to progression (TTP) and overall survival (OS) were 10.0 and 22.0 months, respectively. The treatment was well tolerated, with no treatment-related deaths, while $24.2 \%$ of the patients were affected by cutaneous toxicity of grade $>2$. Mutational analysis of the KRAS and BRAF genes was retrospectively performed on 35 of the 69 patients treated with cetuximab (51\%). KRAS was mutated in 13 out of the 35 cases (37\%), whereas no mutations were detected in the BRAF gene. A trend toward an association between KRAS mutations and objective response to treatment $(p=0.07)$

\section{KARGER}

Fax +41613061234 E-Mail karger@karger.ch www.karger.com (c) 2011 S. Karger AG, Basel

0030-2414/10/0796-0415\$26.00/0

Accessible online at:

www.karger.com/ocl
Giuseppe Colucci, MD

Medical and Experimental Oncology Unit

Cancer Institute 'Giovanni Paolo II', Via Hahnemann

IT-70126 Bari (Italy)

Tel. +39080555 5262, E-Mail colucci@goim.it 
was demonstrated. Analysis of survival showed that patients harboring KRAS mutations had a trend toward worst TTP $(p=0.14)$ confirmed by age- and sex-adjusted Cox multivariate regression (hazard ratio, $\mathrm{HR}=0.62 ; 95 \% \mathrm{Cl}$ : 0.36-1.06; $\mathrm{p}$ $=0.08$ ). Indeed, KRAS mutations were significantly associated with worst OS in both unadjusted analysis ( $p=0.047$; log rank test) and age- and sex-adjusted Cox multivariate regression ( $\mathrm{HR}=0.458 ; 95 \% \mathrm{Cl}: 0.248-0.847 ; \mathrm{p}=0.01)$. Conclusions: These results suggest that the combination of FOLFOX-4 plus cetuximab is very active and obtains long TTP with an acceptable toxicity profile. Indeed, our results are in line with recent findings from phase II and phase III randomized studies providing strong evidence that the efficacy of anti-EGFR $\mathrm{mAb}$ is confined to patients with wild-type KRAS mCRC. Investigation of other predictive biomarkers may be useful to further define the responder population.

Copyright $\odot 2011$ S. Karger AG, Base

\section{Introduction}

Over the last decade, dramatic advances have been made in the treatment of metastatic colorectal cancer (mCRC) due to the introduction of oxaliplatin and irinotecan (CPT-11) into clinical practice. The addition of either drugs to 5-fluorouracil modulated by folinic acid (FU/FA) in the widely used FOLFOX-4 and FOLFIRI regimens increased both the objective response rate (ORR) to $35-50 \%$ and overall survival (OS) up to $18-20$ months [1-3]. Two trials, one French and one Italian, comparing FOLFOX-4 and FOLFIRI, demonstrated the equivalence of the two regimens in terms of ORR, time to progression (TTP) and OS, the main difference being the toxicity profile, with mainly neurotoxicity for FOLFOX and gastrointestinal side effects for FOLFIRI $[2,3]$. The recent addition of biological agents seems to offer a chance of further enhancing the activity of conventional chemotherapy.

Epidermal growth factor receptor (EGFR) is one of the four members of the HER transmembrane tyrosine kinase receptor family. It is overexpressed in a number of solid tumors including CRC. To optimize benefits and reduce the risks as well as contain costs associated with anti-EGFR therapy, EGFR expression has been evaluated as a potential marker of clinical outcome [4]. Nevertheless, it is now well established that response to anti-EGFR monoclonal antibodies (mAb) does not depend upon the level of EGFR expression [5].

Cetuximab (Erbitux ${ }^{\circledR}$ ) is a chimeric mAb targeting the EGFR extracellular domain, thereby preventing its natural ligands from binding and triggering the internal- ization of the receptor [6]. Cumulative data from clinical trials showed an ORR of around $10 \%$ with cetuximab monotherapy in highly pretreated patients $[7,8]$.

In preclinical studies, the combination of oxaliplatin plus cetuximab was clearly more effective in controlling the growth of colon carcinoma xenografts with respect to cetuximab or oxaliplatin monotherapy, emphasizing their synergistic antineoplastic activity [9]. Phase II studies examining the activity and tolerability of cetuximab combined with oxaliplatin and FU/FA have provided interesting results $[10,11]$. To further evaluate this combination, the randomized phase II OPUS (Oxaliplatin and Cetuximab in First-Line Treatment of mCRC) trial compared the efficacy and safety of cetuximab plus FOLFOX-4 with FOLFOX-4 alone as first-line therapy for mCRC [12].

KRAS, the human homolog of the Kirsten rat sarcoma-2 virus oncogene, encodes a small $G$ protein that functions downstream of EGFR-induced cell signaling. Recent clinical data provide growing evidence that KRAS mutational status should be utilized as a molecular marker of anti-EGFR mAb sensitivity in mCRC $[13,14]$. Indeed, mutations of the KRAS gene and valine to glutamic acid substitution (V600E) in the BRAF gene are mutually exclusive events and can constitutively switch on the RAS/ $\mathrm{RAF} /$ mitogen-activated protein kinase pathway [15].

In this study, we evaluated the activity and safety of the combination of cetuximab plus FOLFOX-4 in untreated mCRC patients. Indeed, taking into account the recent evidence that the efficacy of anti-EGFR $\mathrm{mAb}$ is confined to patients with wild-type KRAS mCRC, we retrospectively investigated the influence of KRAS and BRAF status in a subgroup of patients.

\section{Patients and Methods}

Objectives of the Trial

The primary endpoint of the study was to evaluate the activity of this combination in terms of confirmed ORR. Secondary endpoints were: TTP, time to response, and safety of the combination. OS and resectability of liver/lung metastases were also studied.

\section{Patient Selection Criteria}

Patients enrolled in this trial had to satisfy the following criteria: histologically confirmed diagnosis of adenocarcinoma of the colon/rectum; metastatic or locally advanced inoperable disease; the presence of at least 1 measurable lesion (Response Evaluation Criteria in Solid Tumors); expression of EGFR in primary or metastatic tumor detected by immunochemistry; absence of previous chemotherapy or EGFR targeting treatment for advanced disease (adjuvant therapy without oxaliplatin was allowed if recurrence was documented more than 6 months after the end of 
adjuvant treatment); age between 18 and 75 years; Eastern Cooperative Oncology Group performance status of $\leq 2$; adequate bone marrow reserve (neutrophils: $\geq 1,500 / \mathrm{mm}^{3}$; platelets: $\geq 100,000 / \mathrm{mm}^{3}$; hemoglobin level: $\geq 9.0 \mathrm{~g} / \mathrm{dl}$ ); renal and hepatic function (serum creatinine: $<1.5 \times$ upper limit of normal or ULN; transaminases: $<2.5 \times \mathrm{ULN}$, or $<5.0 \times \mathrm{ULN}$ in presence of liver metastases); life expectancy of more than 3 months, and signed written informed consent. Patients were excluded if pretreated with $\mathrm{mAb}$ or signal transduction inhibitors - whether brain metastases, known allergic disease or peripheral neuropathy were present - if they were affected by clinically relevant coronary artery disease or had a history of myocardial infarction. The presence of any concurrent malignancy other than nonmelanoma skin cancer or carcinoma in situ of the cervix was considered an exclusion criterion. The protocol was approved by all ethics committees of the participating institutions.

\section{EGFR Expression}

EGFR expression in the primary tumor and/or metastases was measured using the EGFR pharmDx Kit system (DakoCytomation). Tumor samples were considered positive if EGFR-immunostained cells were more than $1 \%$.

\section{KRAS and BRAF Determinations}

KRAS and BRAF mutational analyses were performed on DNA extracted from paraffin-embedded tissues. For each of the cases, an eosin/hematoxylin-stained section was prepared to ensure that the tumor sample contained more than $70 \%$ tumor cells. DNA was extracted by using the QIAmp DNA Mini Kit (Qiagen, Valencia, Calif., USA) according to standard Clinical Experimental Oncology Laboratory of Bari Quality Certified (DNV No. CERT-17885-2006-AQ-BRI-SINCERT) protocol instructions. PCR amplification was carried out with $100 \mathrm{ng}$ of genomic DNA as a template, $1 \times$ reaction buffer, $1.5-3 \mathrm{mM}$ magnesium chloride, $200 \mu \mathrm{M}$ dNTP, 20-50 pmol of each PCR primer and $0.25 \mathrm{U}$ of AmpliTaq Gold. Mutations in codons 12 and 13 of KRAS were detected by direct sequencing (3130XL; Applied Biosystems) on both strands of 2 independent PCR products, using the following primers: forward 5'-GTA CTG GTG GAG TAT TTG ATA GTG3', and reverse 5'-GGT CAG AGA AAC CTT TAT CTG TAT C-3'. Three different DNA aliquots from the same patient were sequenced.

Mutation in BRAF codon 600 was determined by minisatellite-associated sequence amplification. Two different forward primers with substitution of a single base at the end of the primer (5'-GTGATTTTGGTCTAGCTACAGT-3' and 5'-GTGATTTTGGTCTAGCTACAGA-3') were designed to amplify the wildtype allele or BRAF T1799A transversion mutation, respectively. The reverse primer was common to the 2 reactions $\left(5^{\prime}\right.$-GGCCAAAATTTAATCAGTGGA-3'). The sequence of the reverse primer was the same as used before. The PCR reactions were performed separately in an AppliedBiosystems 9700 Thermal cycler, including an initial denaturation of $2 \mathrm{~min}$ at $94^{\circ} \mathrm{C}$, and a subsequent denaturation for $30 \mathrm{~s}$ at $94^{\circ} \mathrm{C}$, annealing for $30 \mathrm{~s}$ at $58^{\circ} \mathrm{C}$ and extension for $30 \mathrm{~s}$ at $72^{\circ} \mathrm{C}$. PCR reactions were performed in $25 \mu \mathrm{l}$ with $200 \mu \mathrm{M}$ dNTP, $50 \mathrm{ng}$ of genomic DNA, 25 pmol of each primer, $0.5 \mathrm{U}$ of Eppendorf HotMaster Taq and $1 \times$ HotMaster buffer. Thirty-five cycles were used, and the amplification products were subsequently separated on $2.0 \%$ agarose gel and visualized by ethidium bromide staining.

Cetuximab plus FOLFOX-4 in Advanced

Colorectal Cancer Patients

\section{Therapeutic Regimen Administration}

Cetuximab was administered at a starting dose of $400 \mathrm{mg} / \mathrm{m}^{2}$, and then weekly at a dose of $250 \mathrm{mg} / \mathrm{m}^{2}$. The volume of the starting dose was administered over a 2 -hour period (maximum rate of $5 \mathrm{ml} / \mathrm{min}$ ) in a $0.9 \%$ saline solution. Appropriate pretreatment with an antihistaminic drug was mandatory in order to reduce the risk of an allergic reaction. The weekly dose was administered over a 1-hour period. Vital signs were checked before, during and after cetuximab administration as well as $1 \mathrm{~h}$ after infusion to monitor any possible occurrence of adverse events. A regimen of fortnightly FOLFOX-4 infusions [16], commencing $1 \mathrm{~h}$ after the cetuximab infusion had finished, was administered. Premedication with an antiemetic was recommended.

\section{Dose Modification}

The National Cancer Institute Common Toxicity Criteria version 3 were used for grading toxicity. Cetuximab therapy was deferred for up to 2 consecutive infusions with no change in dose level where grade 3 skin toxicity was present. If the toxicity was resolved to grade 2 or less, treatment was resumed at the same dose. With the second and third occurrence of grade 3 skin toxicity, cetuximab therapy was deferred for up to 2 consecutive weeks with concomitant dose reduction to 200 and $150 \mathrm{mg} / \mathrm{m}^{2}$, respectively. The cetuximab dose reduction had to be permanent. Patients discontinued treatment with cetuximab when more than 2 consecutive infusions were observed or there was a fourth occurrence of grade 3 skin toxicity despite an appropriate dose reduction. Dose modification of cetuximab was not permitted for FOLFOX-4-related toxicities. Patients experiencing a grade 3 or 4 anaphylactic reaction had to be discontinued from the study. In the case of FOLFOX-4-related toxicity, where hematological grade 2 toxicities were observed, treatment was delayed for 1 week, maintaining the same dose level. In the presence of grade 3 hematological toxicity, treatment was delayed for 1 week and the dose reduced by 1 level (oxaliplatin: $75 \mathrm{mg} / \mathrm{m}^{2}$; FU bolus: $300 \mathrm{mg} / \mathrm{mm}^{2}$; FU infusion: $500 \mathrm{mg} / \mathrm{m}^{2}$ ). Where grade 4 hematological toxicity was observed, treatment was delayed until the toxicity resolved to grade $\leq 2$, and a reduction in dose level was applied to the following cycles. In the presence of grade 2 diarrhea, 1 dose level reduction was applied. In the presence of grade 3 diarrhea, treatment was delayed for 1 week and resumed with 1 dose level reduction. In grade 4 diarrhea, the treatment was definitely stopped.

\section{Treatment Plan}

Patients had to receive 4 cycles of cetuximab plus FOLFOX-4 prior to the first evaluation of the disease. When a complete remission (CR) or a partial remission (PR) was observed, a confirmatory assessment was performed 4 weeks later. Subsequent disease evaluations were performed every other 4 cycles for a maximum of 12 cycles of chemotherapy. For those patients still on response (CR, PR and stable disease or SD), maintenance with cetuximab alone was continued until progression or inacceptable toxicity for a maximum of 6 months. Evaluation for surgical resection of metastases was performed at study entry as well as after the first confirmed response. Following complete resection, patients were candidates for postsurgery treatment with the same combination for a maximum of $4-6$ cycles. 
Response Evaluation Criteria

Response Evaluation Criteria in Solid Tumors were used for evaluating response to treatment [17]. Time to response was determined for all responders from the first day of therapy until the measurement criteria for objective response were first reported. TTP was calculated for all patients from day 1 of treatment until the date of progression or death; patients who died of disease and for whom a date of progression was not available were considered to have progressed on the day of their death. OS was calculated for all patients from the date of study entry until the date of death or the last follow-up.

\section{Statistical Analysis}

The statistical design was performed according to Simon's one-stage phase II optimal trial design. Assuming $\mathrm{p}_{0}=35 \%$ and $\mathrm{p}_{1}=50 \%$, with an $\alpha$ error $=5 \%$ and a power $\beta=80 \%$, at least 64 patients had to be enrolled. The combination was judged to be active if 29 objective responses were observed.

Data were analyzed after having checked their distribution by using the Kolmogorov-Smirnov and Shapiro tests. Cross tabulation with categorical variables was analyzed by the Fisher exact test or the Mantel-Haenszel $\chi^{2}$ test, whereas comparison between continuous variables was carried out by the Mann-Whitney or Kruskal-Wallis tests. The survival analysis was done with the Kaplan-Meier survivor function followed by the log rank test. Hazard ratios (HR) with their 95\% CI were also estimated using the multivariate Cox proportional hazards model. All analyses were performed using SAS ${ }^{\circledR}$ release 9.1. $\mathrm{p}<0.05$ was considered significant.

\section{Results}

From March 2005 to February 2006, 82 untreated mCRC patients were screened for EGFR. Seventy (85\%) were positive and entered into the trial. Twelve patients were EGFR negative and were excluded. There were 43 males and 27 females, the median age was 62 years (range: 36-74 years), the median pathologic stage was 0 (range: $0-2)$. The primary site of disease was: the colon in 48 cases $(68.6 \%)$ and the rectum in $22(31.4 \%)$. Fifty-three patients (75.7\%) had liver disease, 23 (32.9\%) had lung metastases and 9 (12.9\%) had lymph node disease; 19 patients were affected by disease in other sites (27.1\%). Forty-four patients (63\%) had only 1 site, while $26(37 \%)$ had multiple sites of disease. Fifty-nine patients $(84 \%)$ had synchronous metastases, while only $11(16 \%)$ had metachronous disease. Only 7 patients $(10 \%)$ had received adjuvant treatment. Of the patients with liver involvement alone, 25/33 (76\%) had bulky disease (25-50\% with liver involvement).

Mutational analysis of the KRAS and BRAF genes was retrospectively performed in 35 of the 70 patients treated with cetuximab (50\%). KRAS was mutated in 13 out of
Table 1. Efficacy data (n)

\begin{tabular}{lcc}
\hline Enrolled/screened & $70 / 82(85 \%)$ & \\
Assessable & 67 \\
CR & $4(6 \%)$ & \\
PR & $39(58.2 \%)$ & \\
SD & $20(29.8 \%)$ & \\
PD & $4(6 \%)$ & \\
ORR & $43 / 67(64 \%)$ & $95 \%$ CI: $52.5-75.5$ \\
Disease control rate & $63 / 67(94 \%)$ & $95 \%$ CI: $88-99$ \\
ITT analysis OR & $43 / 70(61 \%)$ & $95 \%$ CI: $50-72$ \\
Disease control rate & $63 / 70(90 \%)$ & $95 \%$ CI: $83-97$ \\
\hline
\end{tabular}

3 patients had no evidence of disease: 2 refused treatment, 1 had an allergic reaction. $\mathrm{PD}=$ Progressive disease; ITT $=$ intention-to-treat; $\mathrm{OR}=$ objective response.

the 35 cases (37\%), whereas no mutations were detected in the BRAF gene. In 11 cases, KRAS mutations were found in codon 12 , and 2 cases had KRAS mutations in codon 13. No BRAF mutations were detected in the tumor analyzed. No association was found between KRAS mutation and age, location of the primary tumor or degree of cutaneous toxicity.

\section{Efficacy}

The results obtained are set out in table 1 . Three patients were not evaluable: 2 due to refusal and 1 because of an allergic reaction after the first cycle of treatment. These 3 patients were also excluded by the KRAS and BRAF analyses. Of the 67 assessable patients, we observed: 4 CR (6\%) and 39 PR (58.2\%) with an ORR of 64.2\% (95\% CI: 52.5-75.5\%), 20 SD (29.8\%) and 4 progressive disease (6\%). The disease control rate was $94 \%$ (95\% CI: 88-99\%). A higher number of responses were observed in patients affected by more severe degrees of cutaneous toxicity. In the group with G0-1 toxicity, the ORR was $43 \%$ (7/16), while in the G3-4 groups the ORR was $70 \%(33 / 47 ; \mathrm{p}=0.08)$. The ORR according to the site of disease were $60 \%$ (32/53 patients) and 52\% (12/23 patients) for liver and lung involvement, respectively. Ten patients (15\%) underwent surgical resection for their metastases. Among the 33 patients with initially unresectable liver disease, 7 (21\%) were resected and a R0 resection was performed on 6 of them. In the group of patients with lung metastases alone, $3 / 6$ (50\%) were radically resected after the treatment. Only 5 patients received the maintenance therapy with cetuximab alone until progression or unacceptable toxicity. Due to the 


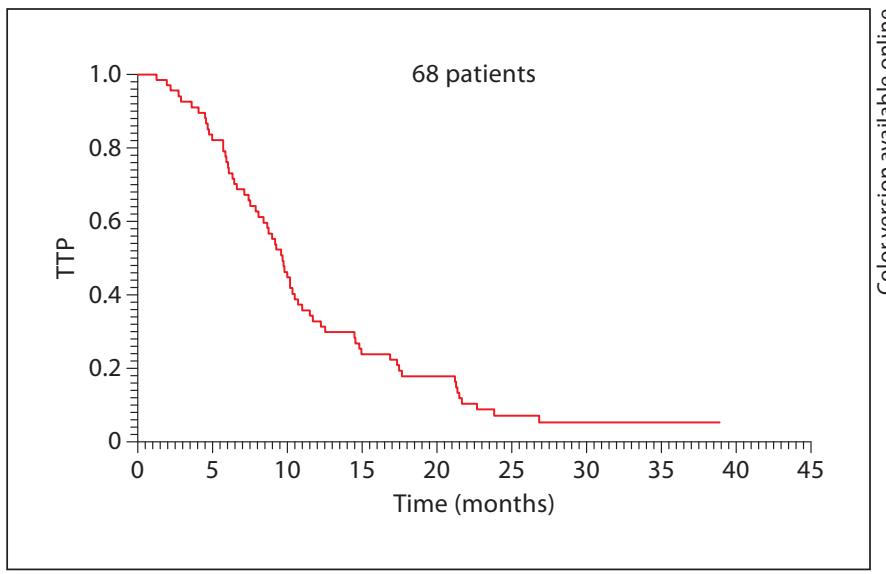

Fig. 1. Kaplan-Meier cumulative progression-free survival for the population cohort.

low number of observations, we did not perform any statistical analysis.

The median TTP and OS were 10.0 and 22.0 months, respectively (fig. 1, 2). The relationship between KRAS mutation and clinical outcome was evaluated in terms of ORR, TTP and OS (table 2). A trend toward an association between KRAS mutation and objective response to treatment ( $\mathrm{p}=0.07$; Mantel-Haenszel test) was demonstrated. In particular, none of the mutated cases showed $\mathrm{CR}$, and only 1 of the wild-type cases progressed. Analysis of survival showed that patients harboring KRAS mutations had a trend toward worst TTP $(\mathrm{p}=0.14$; log rank test ) (fig. 3), confirmed by age- and sex-adjusted Cox multivariate regression $(\mathrm{HR}=0.62 ; 95 \% \mathrm{CI}$ : $0.36-1.06$; $\mathrm{p}=0.08$ ). Indeed, KRAS mutations were significantly associated with worst OS in both unadjusted analysis ( $\mathrm{p}=$ 0.047; log rank test) (fig. 4) and age- and sex-adjusted Cox multivariate regression $(\mathrm{HR}=0.458$; 95\% CI: $0.248-$ $0.847 ; \mathrm{p}=0.01)$. Indeed, in the group of patients that underwent surgical resection for their metastases, we evaluated the KRAS and BRAF status of both primary and metastatic sites. We found a complete concordance of both gene statuses in the 2 sites.

\section{Toxicity}

The median number of delivered courses was 10 (range: 1-12). The combination was well tolerated with no treatment-related deaths. Only 1 patient had a grade 4 allergic reaction and was excluded from the study. The main hematological toxicity was neutropenia, which affected $10 \%$ of the patients, while both anemia and thrombocytopenia were observed in only $1.4 \%$ of cases.

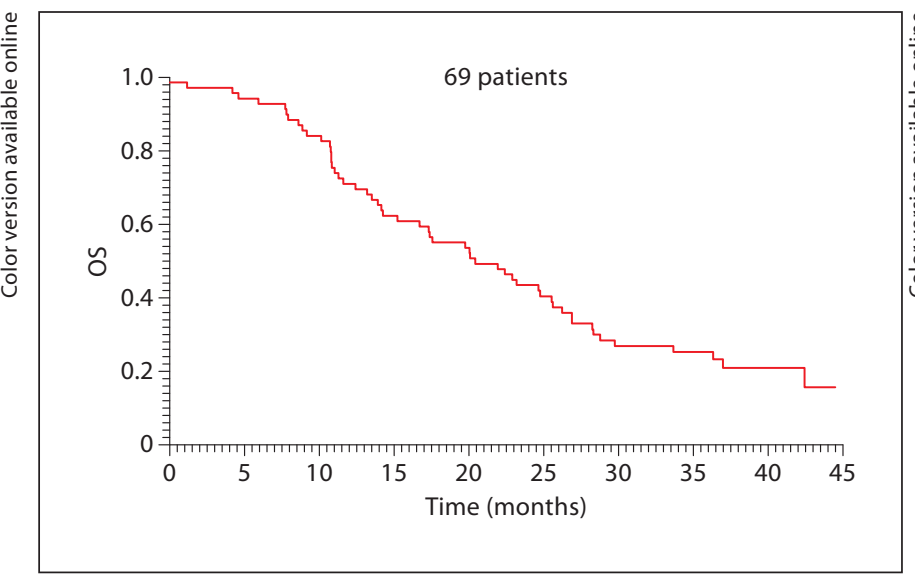

Fig. 2. Kaplan-Meier cumulative OS for the population cohort.

Table 2. Univariate analysis of association of clinical and pathological characteristics with KRAS status

\begin{tabular}{lccc}
\hline & \multicolumn{2}{c}{ KRAS status } & \multirow{2}{*}{$\mathrm{p}$} \\
\cline { 2 - 3 } & WT & Mut & \\
\hline Response to treatment & & & \\
CR & $3(14 \%)$ & $0(0 \%)$ & \\
PR & $13(59 \%)$ & $7(54 \%)$ & \\
SD & $5(23 \%)$ & $3(23 \%)$ & \\
PD & $1(4 \%)$ & $3(23 \%)$ & \\
Cutaneous rash & & & \\
0 & $7(58 \%)$ & $5(38 \%)$ & $0.49^{1}$ \\
1 & $10(59 \%)$ & $7(54 \%)$ & \\
2 & $4(80 \%)$ & $1(8 \%)$ & \\
3 & $0(0 \%)$ & $0(0 \%)$ & $0.03^{2}$ \\
Median TTP, months & $12.0(9.0-17.7)$ & $8.1(4.8-11.0)$ & \\
Median time to death & & & \multirow{2}{*}{$0.11^{2}$} \\
months & $27.3(19.7-36.8)$ & $20.0(12.4-26.9)$ & \\
\hline
\end{tabular}

Values for TTP and time to death in parentheses denote ranges. $\mathrm{WT}=\mathrm{W}$ ild type Mut $=$ mutated $\mathrm{PD}=$ progressive disease

${ }^{1}$ Mantel-Haenszel test. ${ }^{2}$ Mann-Whitney test.

Nonhematological toxicity was mainly gastrointestinal, with mucositis, diarrhea and nausea/vomiting observed in $8.6 \%$ of the patients. Neurotoxicity affected $40 \%$ of the patients, but it was of grade $3-4$ in only $2.9 \%$ of the cases. As expected, cutaneous toxicity was the most relevant side effect, involving 64 patients (91.3\%), and it was recorded as grade 3-4 in 17 patients (24.2\%) (table 3). 


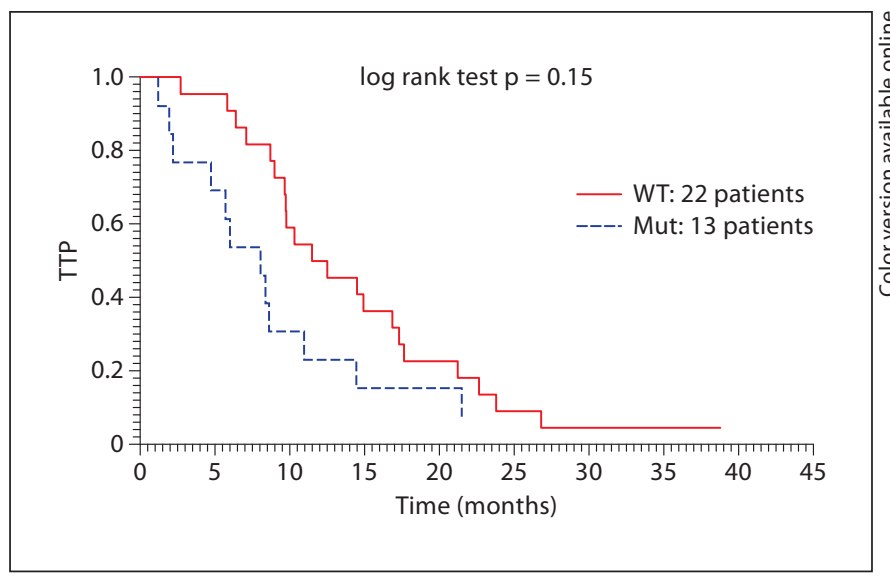

Fig. 3. Kaplan-Meier cumulative progression-free survival on the basis of KRAS mutational status. Wt = Wild type; Mut = mutated.

Table 3. National Cancer Institute toxicity criteria

\begin{tabular}{lcccc}
\hline & $\begin{array}{l}\text { Patients } \\
\mathrm{n}\end{array}$ & $\begin{array}{l}\text { G1-2 } \\
\%\end{array}$ & $\begin{array}{l}\text { Patients } \\
\mathrm{n}\end{array}$ & $\begin{array}{l}\mathrm{G} 3-4 \\
\%\end{array}$ \\
\hline Leukopenia & 4 & 5.7 & 5 & 7.1 \\
Neutropenia & 3 & 4.3 & 7 & 10 \\
Thrombocytopenia & 9 & 12.9 & 1 & 1.4 \\
Anemia & 16 & 22.8 & 1 & 1.4 \\
Nausea/vomiting & 30 & 42.8 & 6 & 8.6 \\
Diarrhea & 24 & 34.3 & 6 & 8.6 \\
Mucositis & 11 & 15.7 & 6 & 8.6 \\
Fever & 7 & 10 & 2 & 2.9 \\
Loss of hair & 11 & 15.7 & - & - \\
Neurological & 26 & 37.1 & 2 & 2.9 \\
Cutaneous & 47 & 67.1 & 17 & 24.2 \\
Asthenia & 15 & 21.4 & - & - \\
Stypsis & 6 & 8.6 & - & - \\
Hepatic & 7 & 10 & 1 & 1.4 \\
Allergic reaction & - & - & 1 & 1.4 \\
\hline
\end{tabular}

Table 4. Cetuximab-based regimens as neoadjuvant treatments of liver metastases

\begin{tabular}{lll}
\hline Study & Schedule & $\begin{array}{l}\text { Resectability } \\
\text { rate, \% }\end{array}$ \\
\hline Folprecht et al. [23] & FOLFIRI + cetuximab & 19 \\
Van Cutsem et al. [25] & FOLFIRI + cetuximab & 9.8 \\
Tabernero et al. [10] & FOLFOX-4 + cetuximab & 19 \\
Bokemeyer et al. [12] & FOLFOX-4 + cetuximab & 6.5 \\
Present study & FOLFOX-4 + cetuximab & 21 \\
\hline
\end{tabular}

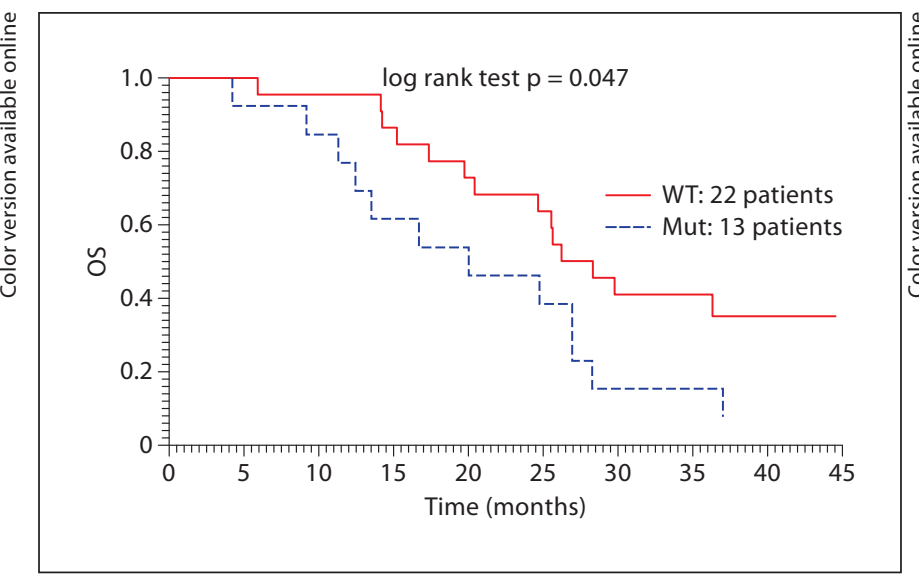

Fig. 4. Kaplan-Meier cumulative OS on the basis of KRAS mutational status. WT $=$ Wild type; Mut $=$ mutated.

\section{Discussion}

In our study, we observed a high activity of the combination of cetuximab plus FOLFOX-4 in untreated mCRC patients with an ORR of $64 \%$ and a disease control rate of $94 \%$ in assessable patients. These results are even more interesting because they were obtained in an unselected population having unfavorable prognostic factors such as a high predominance of synchronous metastases and the presence of multiple sites of disease, affecting 84 and 37\% of the patients, respectively. Other trials employing cetuximab plus oxaliplatin-based schedules obtained similar results. Tabernero et al. [10], in 43 patients treated with our combination, reported an ORR of $72 \%$ and a tumor growth control rate (TGCR) of $95 \%$. However, it should be noted that, in this trial, a large number of patients had a low disease burden, with $47 \%$ having only 1 single metastatic site. Dakhil et al. [18], employing a cetuximab plus modified FOLFOX regimen, observed an ORR of $61 \%$ and a disease control rate of about $90 \%$ in 67 patients, while Seufferlein et al. [19], employing cetuximab plus oxaliplatin and FU/FA administered according to an AIO (Arbeitsgemeinschaft Internistische Onkologie) regimen, reported an ORR of 54\% in 41 patients. Also, in the redesigned randomized phase II trial CALGB 80203 study, in 58 patients treated with the same combination employed in our study, the ORR was $60 \%$ and the disease control rate was $86 \%$ [20], and in the OPUS phase II randomized trial, the observed ORR in 169 patients was 46\% [12]. These differences can probably be ascribed to patient selection for small phase II studies compared with larger studies that recruit unselected patients. 
If we consider the median TTP and OS, the values of 10.0 and 22.0 months obtained in the current trial are lower than those reported by Tabernero et al. [10], but this difference is probably due to the selection of patients in the two studies. Considering the median TTP and OS observed in our previous study testing FOLFOX-4 and FOLFIRI head-to-head [3], the addition of cetuximab to FOLFOX-4 seems to offer an incremental benefit compared to FOLFOX- 4 alone (10.0 vs. 7.0 and 22.0 vs. 15 months, respectively), although the limitations of a phase II trial should be taken into account.

Another concept that needs to be stressed is the possibility of employing a combination capable of obtaining a high percentage of ORR as a neoadjuvant treatment for isolated liver or lung involvement. The liver is the most common site of metastases in $\mathrm{mCRC}$ and the prognosis of initially resectable patients is similar to those of patients downstaged and resected after chemotherapy [21, 22]. The main predictor of resectability is antitumor activity measured in terms of its ORR [23]. In our study, 53 (75\%) of our unselected patients had liver involvement, and the liver was the only site of disease in 33 of them. Seven out of 33 (21\%) with initially unresectable disease proved suitable for resection after treatment and a $\mathrm{R} 0$ resection was obtained in 6 of them. The OS for these patients was $18,22,24+, 26+, 27+, 28+$ and $28+$ months. Data in the literature indicate that, in unselected series, the percentage of initially unresectable liver metastases in patients who underwent surgery after shrinkage of disease ranges between 1 and $26 \%$ (table 4 ). In particular, Tabernero et al. [10] reported $21 \%$ of initially unresectable patients who had undergone surgery after chemotherapy (19\% of them had liver disease alone). Our data confirm this possibility, especially in view of the short response time of approximately 2.5 months, which could prevent liver damage from prolonged treatment [24]. With regard to toxicity, the combination of cetuximab plus FOLFOX-4 was well tolerated. No treatment-related deaths were observed and only 1 patient was excluded from the study because of an allergic reaction. The addition of cetuximab to FOLFOX did not increase the toxicity related to the chemotherapy alone. Grade 3-4 hematological toxicity was limited, the main side effect being neutropenia in $10 \%$ of the cases, while grade 3-4 nonhematological toxicities were nausea/vomiting, diarrhea and mucositis, affecting less than $10 \%$ of patients. Grade 3-4 neurotoxicity was observed in about 3\% of the patients. With regard to cutaneous toxicity, the reported $24 \%$ of grade 3-4 side effects is similar to the results of other series: Tabernero et al. [10] and Dakhil et al. [18] reported 30 and $17 \%$ of cutaneous toxicity, respectively. In our series, we observed a difference in terms of ORR according to skin toxicity (G0-1: 43\%; G3-4: 70\%), even if the low number of patients could explain the lack of statistical significance $(\mathrm{p}=0.08)$.

In conclusion, in our study the addition of cetuximab to the FOLFOX-4 regimen achieved high response rates with a good safety profile in mCRC patients; these results translated into an interesting percentage of resectability for initially unresectable liver metastases. Thus it appears possible to employ this combination as a front-line therapy, mainly in patients with potentially resectable disease as the ultimate goal in this context is cure.

When retrospective efficacy analyses were performed according to KRAS mutation status, differences were apparent in terms of ORR and survival, even if the low number of patients could be responsible of the lack of statistical significance. In particular, patients whose tumors were wild-type KRAS showed a trend toward a better ORR, with only 1 of the wild-type cases progressed. Similarly, analysis of survival showed that tumors harboring KRAS mutations had a trend toward worst TTP. These results are in line with recent findings from phase II [13] and phase III randomized studies [25], providing strong evidence that the efficacy of anti-EGFR $\mathrm{mAb}$ is confined to patients with wild-type KRAS mCRC, and that genotyping of tumors should be considered in these patients before treatment with these drugs. According to these results, approval for cetuximab is actually restricted to mCRC patients with wild-type KRAS tumors. Finally, the dimension of the cases should explain the absence of BRAF mutations. In the near future, an investigation of other biomarkers may be useful to further define the responder population.

\section{Disclosure Statement}

The authors have indicated no financial relationships with companies whose products are mentioned in this article. 


\section{References}

1 Kelly H, Goldberg RM: Systemic therapy for metastatic colorectal cancer: current options, current evidence. J Clin Oncol 2005; 23:4553-4560.

2 Tournigand C, André T, Achille E, Lledo G, Flesh M, Mery-Mignard D, Quinaux E, Couteau C, Buyse M, Ganem G, Landi B, Colin P, Louvet C, de Gramont A: FOLFIRI followed by FOLFOX- 6 or the reverse sequence in advanced colorectal cancer: a randomized GERCOR study. J Clin Oncol 2004;22:229237.

3 Colucci G, Gebbia V, Paoletti G, Giuliani F, Caruso M, Gebbia N, Cartenì G, Agostara B, Pezzella G, Manzione L, Borsellino N, Misino A, Romito S, Durini E, Cordio S, di Seri M, Lopez M, Maiello E, Montemurro S, Cramarossa A, Lorusso V, di Bisceglie M, Chiarenza M, Valerio MR, Guida T, Leonardi V, Pisconti S, Rosati G, Carrozza F, Nettis G, Valdesi M, Filippelli G, Fortunato S, Mancarella S, Brunetti C: Phase III randomized trial of FOLFIRI versus FOLFOX-4 in the treatment of advanced colorectal cancer: a multicenter study of the Gruppo Oncologico dell'Italia Meridionale. J Clin Oncol 2005; 23:4866-4875.

4 Wells A: EGF receptor. Int J Bioch Cell Biol 1999;31:637-643.

5 Chung KY, Shia J, Kemeny NE, Shah M, Schwartz GK, Tse A, Hamilton A, Pan D, Schrag D, Schwartz L, Klimstra DS, Fridman D, Kelsen DP, Saltz LB: Cetuximab shows activity in colorectal cancer patients with tumors that do not express the epidermal growth factor receptor by immunohistochemistry. J Clin Oncol 2005;23:1803-1810.

6 Ciardiello F, Tortora G: EGFR antagonists in cancer treatment. N Engl J Med 2008;358: $1160-1174$.

7 Saltz L, Meropol NJ, Loehrer PJ, Needle MN, Kopit J, Mayer RJ: Phase II trial of cetuximab in patients with refractory colorectal cancer that expresses the epidermal growth factor receptor. J Clin Oncol 2004;22:1201-1208.

8 Lenz HJ, van Cutsem E, Khambata-Ford S, Mayer RJ, Gold P, Stella P, Mirtsching B, Cohn AL, Pippas AW, Azarnia N, Tsuchihashi Z, Mauro DJ, Rowinsky EK: Multicenter phase II and translational study of cetuximab in metastatic colorectal carcinoma refractory to irinotecan, oxaliplatin, and fluoropyrimidines. J Clin Oncol 2006;24:49144921.

9 Prewett M, Deevi DS, Bassi R, Fan F, Ellis LM, Hicklin DJ, Tonra JR: Tumors established with cell lines selected for oxaliplatin resistance respond to oxaliplatin if combined with cetuximab. Clin Cancer Res 2007; 13:7432-7440.
10 Tabernero J, van Cutsem E, Díaz-Rubio E, Cervantes A, Humblet Y, André T, van Laethem JL, Soulié P, Casado E, Verslype C, Valera JS, Tortora G, Ciardiello F, Kisker O, de Gramont A: Phase II trial of cetuximab in combination with fluorouracil, leucovorin, and oxaliplatin in the first-line treatment of metastatic colorectal cancer. J Clin Oncol 2007;25:5225-5232.

11 Arnold D, Höhler T, Dittrich C, Lordick F, Seufferlein T, Riemann J, Wöll E, Herrmann T, Zubel A, Schmoll HJ: Cetuximab in combination with weekly 5 -fluorouracil/folinic acid and oxaliplatin (FUFOX) in untreated patients with advanced colorectal cancer: a phase Ib/II study of the AIO GI Group. Ann Oncol 2008;19:1442-1449.

12 Bokemeyer C, Bondarenko I, Makhson A, Hartmann JT, Aparicio J, de Braud F, Donea S, Ludwig H, Schuch G, Stroh C, Loos AH, Zubel A, Koralewski P: Fluorouracil, leucovorin, and oxaliplatin with and without cetuximab in the first-line treatment of metastatic colorectal cancer. J Clin Oncol 2009; 27:663-671.

13 Silvestris N, Tommasi S, Santini D, Russo A, Simone G, Petriella D, Maiello E, Tonini G, Colucci G: KRAS mutations and sensitivity to anti-EGFR monoclonal antibodies in metastatic colorectal carcinoma: an open issue. Expert Opin Biol Ther 2009;9:565-577.

14 Allegra CJ, Jessup JM, Somerfield MR, Hamilton SR, Hammond EH, Hayes DF, McAllister PK, Morton RF, Schilsky RL: American Society of Clinical Oncology provisional clinical opinion: testing for KRAS gene mutations in patients with metastatic colorectal carcinoma to predict response to anti-epidermal growth factor receptor monoclonal antibody therapy. J Clin Oncol 2009;27: 2091-2096.

15 di Nicolantonio F, Martini M, Molinari F Sartore-Bianchi A, Arena S, Saletti P, de Dosso S, Mazzucchelli L, Frattini M, Siena S, Bardelli A: Wild-type BRAF is required for response to panitumumab or cetuximab in metastatic colorectal cancer. J Clin Oncol 2008;26:5705-5712.

16 de Gramont A, Figer A, Seymour M, Homerin M, Hmissi A, Cassidy J, Boni C, CortesFunes H, Cervantes A, Freyer G, Papamichael D, le Bail N, Louvet C, Hendler D, de Braud F, Wilson C, Morvan F, Bonetti A: Leucovorin and fluorouracil with or without oxaliplatin as first-line treatment in advanced colorectal cancer. J Clin Oncol 2000; 18:2938-2947.

17 Therasse P, Arbuck SG, Eisenhauer E: New guidelines to evaluate the response to treatment in solid tumors. J Natl Cancer Inst 2000;92:205-216.
18 Dakhil S, Cosgriff T, Headley D, et al: Cetuximab + FOLFOX-6 as first-line therapy for metastatic colorectal cancer. An International Oncology Network study, I-03-002 (abstract 3557). J Clin Oncol 2006;24(18 suppl):3557a.

19 Seufferlein T, Dittrich C, Riemann JF, Woell E, Herrmann T, Lordick F, Arnold D, Olchowka K, Höhler T, Schmoll HJ: A phase I/II study of cetuximab in combination with 5-fluorouracil (5-FU)/folinic acid (FA) plus weekly oxaliplatin (L-OHP) (FUFOX) in the first-line treatment of patients with metastatic colorectal cancer (mCRC) expressing epidermal growth factor receptor (EGFR): preliminary results (abstract 3644). J Clin Oncol 2005;23(16 suppl):3644a.

20 Venook A, Niedzwiecki D, Hollis D, Sutherland S, Goldberg R, Alberts S, Benson A, Wade J, Schilsky R, Mayer R: Phase III study of irinotecan/5FU/LV (FOLFIRI) or oxaliplatin/5FU/LV (FOLFOX) \pm cetuximab for patients (pts) with untreated metastatic adenocarcinoma of the colon or rectum (MCRC): CALGB 80203 preliminary results. J Clin Oncol 2006;24(18 suppl):3509a.

21 Jamison RL, Donohue JH, Nagorney DM, Rosen CB, Harmsen WS, Ilstrup DM: Hepatic resection for metastatic colorectal cancer results in cure for some patients. Arch Surg 1997;132:505-510.

22 Adam R, Delvart V, Pascal G, Valeanu A, Castaing D, Azoulay D, Giacchetti S, Paule B, Kunstlinger F, Ghémard O, Levi F, Bismuth $\mathrm{H}$ : Rescue surgery for unresectable colorectal liver metastases downstaged by chemotherapy: a model to predict long-term survival. Ann Surg 2004;240:644-658.

23 Folprecht G, Grothey A, Alberts S, Raab HR, Köhne $\mathrm{CH}$ : Neoadjuvant treatment of unresectable colorectal liver metastases: correlation between tumor response and resection rates. Ann Oncol 2005;16:1311-1319.

24 Aloia T, Sebagh M, Plasse M, Karam V, Lévi F, Giacchetti S, Azoulay D, Bismuth H, Castaing D, Adam R: Liver histology and surgical outcomes after preoperative chemotherapy with fluorouracil plus oxaliplatin in colorectal cancer liver metastases. J Clin Oncol 2006;24:4983-4990.

25 van Cutsem E, Köhne CH, Hitre E, Zaluski J, Chang Chien CR, Makhson A, D'Haens G, Pintér T, Lim R, Bodoky G, Roh JK, Folprecht G, Ruff P, Stroh C, Tejpar S, Schlichting M, Nippgen J, Rougier P: Cetuximab and chemotherapy as initial treatment for metastatic colorectal cancer. N Engl J Med 2009;360: $1408-1417$. 